\title{
Type-IIB Instanton as a Wave in Twelve Dimensions
}

\author{
A. A. Tseytlin *,† \\ Theory Division, CERN, CH-1211, Geneve 23, Switzerland \\ and Blackett Laboratory, Imperial College, London, SW7 2BZ, United Kingdom
}

(Received 19 December 1996)

\begin{abstract}
0-brane of type-IIA string theory can be interpreted as a dimensional reduction of a gravitational wave in 11 dimensions. We observe that a similar interpretation applies also to the $D$-instanton background of type-IIB theory: It can be viewed as a reduction (along one spatial and one timelike direction) of a wave in a 12-dimensional theory. The instanton charge is thus related to a linear momentum in 12 dimensions. This suggests that the instanton should play as important a role in type-IIB theory as the 0-brane is supposed to play in type-IIA theory. [S0031-9007(97)02576-3]
\end{abstract}

PACS numbers: 11.25.Mj, 11.15.Tk, 11.30.-j

Recent progress in fundamental string theory has led to important insights about the role of extended solitons ( $p$-branes) and nonperturbative (strong coupling-weak coupling) duality symmetries relating various string vacua [1]. The standard superstring models are approximated at low energies by field theories of 10-dimensional supergravities, containing in their spectrum the graviton, gravitino, as well as several scalars, antisymmetric tensors, and fermions. It is now believed that the previously known superstring models appear as "phases" of more basic theory ( $M$ theory) whose lowenergy limit is described by 11-dimensional supergravity. Understanding the nonperturbative structure of the corresponding quantum theory is crucial in order to be able to explain how string theory is related to the 4-dimensional world upon Kaluza-Klein-type compactification of extra 'internal' dimensions.

As was known for more than a decade, there are two consistent closed superstring models with a maximal amount of supersymmetry in ten dimensions, the type-IIA and type-IIB theories [2]. Their low-energy limits are represented by the extended $N=2$ supergravities in 10 dimensions having the Majorana-Weyl supercharges of opposite (IIA) or equal (IIB) chirality. The elementary field content and the spectrum of extended $p$-brane solitons of type-IIA supergravity are directly related to the content of the maximal 11-dimensional supergravity via dimensional reduction in circular 11th dimension. As was argued in [3], the radius of the 11th dimension is related to the type-IIA string coupling constant, so that the strong coupling limit of the type-IIA theory should be uncompactified 11-dimensional $M$ theory [3,4]. When one of the nine spatial dimensions is compactified on a circle, the type-IIA and type-IIB string theories become equivalent by perturbative " $T$ duality." However, in general, the existence of type-IIB string theory not directly related to 11-dimensional $M$ theory seems to be suggesting that there may be a more fundamental picture, in which the type-IIA (and related $M$ theory) and type-IIB models appear more symmetrically.
The presence of the intrinsic $\operatorname{SL}(2, R)$ symmetry [5] of $D=10$ type-IIB supergravity hints at a 12-dimensional reformulation of this theory (compactification of the two extra ficticious dimensions would then explain the symmetry in 10 dimensions). This could lead to a nonperturbative formulation of a type-IIB superstring theory. There were several suggestions in this direction [6-8]. Though no standard supergravity theory is expected to exist in 12 dimensions [yet there is a candidate superalgebra in the space with signature $(10,2)$ [9]] there may be some constrained unconventional theory which regains the Lorentz and diffeomorphism invariance upon reduction to 10 dimensions. Such a 12-dimensional theory may also shed new light on the role and place of 11-dimensional $M$ theory.

It was suggested in $[4,10]$ that 0 -branes [11] may be considered as basic building blocks of $M$ theory. This is related to the fact that the 0-brane charge can be interpreted as a 11-dimensional momentum. At the level of the classical solutions, the $D=100$-brane background is just a dimensional reduction of a gravitational wave propagating in 11 dimensions, $d s_{11}^{2}=-d t^{2}+d x_{11}^{2}+$ $\left(q / r^{7}\right)\left(d t-d x_{11}\right)^{2}+d x_{i} d x_{i}$. The fundamental nature of the 0-brane is also indicated by the fact that other extended objects in the theory can be "constructed" out of arrays of 0-branes by duality transformations.

It seems important to understand the type-IIB theory analog of this picture. The object of minimal dimensionality here is the $D$-instanton [11-13]. It is related to $D 0$-brane by formal $T$ duality in the time direction. This is a hint that the instanton should play a central role in (a 12-dimensional reformulation of) type-IIB theory. In fact, the recent proposal of a matrix model behind type-IIB theory [14], which is based on a large $N$ limit of the zero-dimensional reduction of $\mathrm{SU}(N)$ 10D super Yang-Mills theory, may be interpreted in this way: $\left(L=\operatorname{tr}\left(\left[A_{\mu}, A_{\nu}\right]^{2}+2 i \bar{\psi} \gamma^{\mu}\left[A_{\mu}, \psi\right]\right)\right.$ is the leading term in the action for $N D$-instantons [15]). (In view of $T$ duality between instantons and 0-branes, it is not surprising that this action can be formally related [14] to the 
0-brane action used in [10]. A similar action, but in 12 dimensions, was suggested in connection with the action of [10] in [16].) Below we shall provide new evidence of a fundamental nature of the type-IIB instanton, and, at the same time, of an existence of a 12-dimensional structure behind type-IIB theory: Just as the type-IIA 0 -brane corresponds to a gravitational wave in $11 \mathrm{di}$ mensions, the type-IIB instanton is an "image" of a gravitational wave in 12 dimensions. In particular, the instanton charge is thus identified with the 12-dimensional momentum. Our discussion of the 12-dimensional interpretation of the type-IIB instanton solution of [17] (see also [18]) will be in the spirit of the $F$ theory proposals in [6] and, especially, in [7]. (For other suggestions about the 12-dimensional origin of type-IIB theory, see [8].) There will be an important new point: We will need to consider the Euclidean version of type-IIB theory [with signature $(0,10)]$, and thus will compactify a $D=12$ theory with signature $(1,11)$ on a 2 -space of $(1,1)$ signature.

While a hypothetical 12-dimensional theory which leads to type-IIB theory upon dimensional reduction cannot be of the standard supergravity type, it may not be that different, assuming certain additional constraints are imposed. The $\operatorname{SL}(2, R)$ structure of the field equations [5] or the action [19] of type-IIB theory provides strong hints about its 12-dimensional counterpart. Let us first consider the type-IIB $(1,9)$ theory with a Minkowski signature. The 12-dimensional action should contain at least the Einstein term, probably supplemented with certain conditions on the $D=12$ metric. We shall adopt the following ansatz for the metric $(\mu, \nu=0,1, \ldots, 9 ; p, q=1,2)$ :

$$
\begin{aligned}
d s_{12}^{2}= & d s_{10 E}^{2}+d s_{2}^{2}=g_{\mu \nu}(x) d x^{\mu} d x^{\nu}+M_{p q}(x) d y^{p} d y^{q} \\
= & g_{\mu \nu}(x) d x^{\mu} d x^{\nu}+e^{-\phi(x)} d y_{1}^{2} \\
& +e^{\phi(x)}\left[d y_{2}+C(x) d y_{1}\right]^{2}
\end{aligned}
$$

where $g_{\mu \nu}, \phi$ and $C$ are the Einstein-frame metric, dilaton, and $R-R$ scalar of IIB theory. The metric $M$ of an internal 2-torus with the complex structure modulus $\tau$ is

$$
\begin{aligned}
M_{p q} & =e^{\phi}\left(\begin{array}{cc}
e^{-2 \phi}+C^{2} & C \\
C & 1
\end{array}\right), \quad \tau=C+i e^{-\phi}, \\
\operatorname{det} M_{p q} & =1
\end{aligned}
$$

Note that in contrast to the similar relation between the $D=11$ supergravity and type IIA theory metrics, i.e., $d s_{11}^{2}=e^{-\phi / 6} d s_{10 E}^{2}+e^{4 \phi / 3}\left(d y+A_{\mu} d x^{\mu}\right)^{2}$, the ansatz (1) is not of the most general type: The volume of the internal 2-torus is assumed to be nondynamical $(\operatorname{det} M=$ 1) [7] as there are only two scalars in type-IIB action. Dimensional reduction then gives

$$
\begin{aligned}
S & =\int d^{12} x \sqrt{g_{(12)}} R_{(12)} \\
& =\int d^{10} x \sqrt{g}\left[R+\frac{1}{4} \operatorname{Tr}\left(\partial_{\mu} M \partial^{\mu} M^{-1}\right)\right], \\
= & \int d^{10} x \sqrt{g}\left[R-\frac{1}{2} \tau_{2}^{-2}|\partial \tau|^{2}\right] \\
= & \int d^{10} x \sqrt{g}\left[R-\frac{1}{2}(\partial \phi)^{2}-\frac{1}{2} e^{2 \phi}(\partial C)^{2}\right] .
\end{aligned}
$$

The metric (1) and the action (3) are covariant under the $\operatorname{SL}(2, R)$, explaining the corresponding symmetry of type-IIB theory [6,7]. Other terms in the bosonic part of the type-IIB action can be understood by making a bold assumption that the $D=12$ theory should contain also the 3-rank and 4-rank antisymmetric tensors $C_{3}$ and $C_{4}$. Then the two 2-rank tensors of type-IIB theory $B_{p}(p=$ 1,2) appear as $C_{\mu \nu p}$ components of $C_{3}$, and, moreover, the natural kinetic term $F^{2}\left(C_{3}\right)$ reduces to another important $\operatorname{SL}(2, R)$ covariant structure in the type-IIB action, $M^{p q} d B_{p} d B_{q}$. The $D=12$ Chern-Simons coupling $\int C_{4} \wedge d C_{3} \wedge d C_{3}$ produces the $\int C_{4} \wedge d B^{(1)} \wedge d B^{(2)}$ term in IIB theory action [20]. Obviously, there should be other constraints that should (i) rule out extra terms which appear from $\int\left[R_{(12)}-F^{2}\left(C_{3}\right)-F^{2}\left(C_{4}\right)-C_{4} \wedge\right.$ $\left.d C_{3} \wedge d C_{3}+\cdots\right]$ upon direct dimensional reduction, (ii) imply self-duality of the field strength of $C_{4}$ in $D=10$, and, of course, (iii) ensure supersymmetry.

Assuming the existence of such 12-dimensional theory, it should be possible to relate type-IIB $p$-brane solutions to certain 12-dimensional field configurations. It is natural to expect that the $D=12$ theory should have 3-brane and 5-brane solutions (which are "electromagnetic" dual in $D=12)$. The $\operatorname{SL}(2, Z)$ family of typeIIB strings [21] then may appear as wrappings of the 12-dimensional 3-brane around the internal 2-torus. To understand such a relation in detail one first needs to clarify the structure of the antisymmetric tensor field couplings in the $D=12$ theory. [Naive wrapping of 3-brane with $C_{4}$ charge does not seem to give the charges of the $B_{p}$ fields related to $C_{3}$ as discussed above. One also needs to understand how to connect the $\operatorname{SL}(2, Z)$ family of type-IIB 5-branes to the $D=12$ 5-brane: A puzzle here is that the internal 2-torus should not be part of the 5-brane (see also below).]

Below we shall concentrate on purely gravitational $D=12$ backgrounds which do not depend on unknown details of the structure of the antisymmetric tensor sector. Like the 0-brane and the 6-brane of type-IIA theory which correspond to the purely gravitational solutions in $D=11$ theory (plane wave and Euclidean Taub-NUT or Kaluza-Klein monopole), the instanton and the 7-brane of type-IIB theory also have purely gravitational counterparts in $D=12$. The 7-brane case was already discussed in [7]. The solution corresponding to a collection of $n$ 
type-IIB 7-branes [17,22] is given by (1) of the form,

$$
\begin{aligned}
d s_{12}^{2}= & -d t^{2}+d x_{1}^{2}+\cdots+d x_{7}^{2}+H^{2}(z, \bar{z}) d z d \bar{z} \\
& +H^{-1}(z, \bar{z})\left|d y_{2}+\tau(z) d y_{1}\right|^{2},
\end{aligned}
$$

where $z=x_{8}+i x_{9}, \quad H=e^{-\phi}=\tau_{2}$, and $j[\tau(z)]=$ $P_{n}(z) / P_{n-1}(z)$. The regular case of $n=24$ 7-branes on a compact $(z, \bar{z}) 2$-space in type-IIB theory can be interpreted [7] as a special K3 compactification [22] of the 12-dimensional theory.

Our aim here is to give a similar interpretation to the type-IIB $D$-instanton. The instanton is a solution [17] of the Euclidean type-IIB theory (which has a welldefined Euclidean supersymmetry) with the action (4), where $g_{\mu \nu}$ is assumed to have a Euclidean signature and the scalar $C$ is replaced by $i C$. This rotation of $C$ has a $D=10$ explanation if type-IIB theory is defined in terms of the dual $F_{9}$ field strength [17]. At the same time, it has also an alternative natural $D=12$ explanation if the Euclidean type-IIB $(0,10)$ theory corresponds to a compactification of the same 12-dimensional theory of the signature $(1,11)$, but now on a 2 -space of the signature $(1,1)$. If $g_{\mu \nu}$ in (1) is taken to be Euclidean, $y_{1}$ should become timelike, $y_{1}=-i t$. To preserve the reality of the metric (1) one should then rotate $C \rightarrow i C$. The result is $\left(y \equiv y_{2} ; m, n=1,2, \ldots, 10\right)$

$$
\begin{aligned}
d s_{12}^{2}= & -e^{-\phi(x)} d t^{2}+e^{\phi(x)}[d y+C(x) d t]^{2} \\
& +g_{m n}(x) d x^{m} d x^{n} .
\end{aligned}
$$

The type-IIB ${ }_{(0,10)}$ theory is then obtained by dimensional reduction in the spatial direction $y$ and the timelike direction $t$ (cf. [7,8]). One of the simplest examples of such a gravitational background is a spherically symmetric $p p$ wave,

$$
\begin{aligned}
d s_{12}^{2} & =-d t^{2}+d y^{2}+[H(x)-1](d t-d y)^{2}+d x_{m} d x_{m} \\
& =-H^{-1} d t^{2}+H\left[d y+\left(H^{-1}-1\right) d t\right]^{2}+d x_{m} d x_{m}, \quad H=1+\frac{q}{x^{8}}
\end{aligned}
$$

It solves the vacuum Einstein equations and should be supersymmetric (as is always the case in lower dimensions), provided a supersymmetric $D=12$ theory can be defined. Comparing (6) and (7), we learn that the corresponding type-IIB $(0,10)$ background is exactly the instanton solution of [17]

$$
\begin{aligned}
e^{\phi} & =H(x), \quad C=H^{-1}(x)-1, \\
d s_{10 E}^{2} & =d x_{m} d x_{m} .
\end{aligned}
$$

We assumed that the fields have trivial asymptotic values $\phi_{\infty}=0, C_{\infty}=0$, i.e., that the vacuum "2-torus" $T^{(1,1)}$ is trivial. The analog of the $\operatorname{SL}(2, R)$ symmetry in typeIIB $_{(0,10)}$ theory acts only on the constant parameters $(g=$ $\left.e^{\phi \infty}, C_{\infty}\right)$ of the generic solution. The constant $q$ is related to the instanton charge $Q_{-1}=8 \omega_{9} q=\frac{2}{3} \pi^{5 / 2} q$ [17], which can now be interpreted as a linear momentum carried by the wave in the 12th dimension. As in the case of the "0-brane charge-11-dimensional momentum" correspondence, this provides another reason for the quantization of $Q_{-1}$.

Let us now discuss some implications of the above observation. Suppose that the metric (7) has an extra spatial isometry in one of the $x_{m}$ directions, e.g., in $x_{10} \equiv z$ (then $\left.H=1+q / x^{7}\right)$. The reduction along $(t, y)$ then connects it to a type-IIB $(0,10)$ background produced by a periodic array of instantons in $z$ direction. If we also assume that the 12-dimensional theory is somehow related to $M$ theory by a reduction in $z$ [more generally, that a compactification of the $(2,10)$ theory on $T^{(1,1)} \times S^{1}$ corresponds to a compactification of $(1,10)$ theory on $S^{1} \times$ $S^{1}$, cf. [7]], then the resulting 11-dimensional background is again a similar plane wave. Further reduction along $y$ leads to the 0-brane solution of type-IIA theory with $d s^{2}=$ $H^{1 / 2}\left(-H^{-1} d t^{2}+d x_{k} d x_{k}\right), e^{\phi}=H^{3 / 4}, A_{t}=H^{-1}-1$. This is related to the above type-IIB ${ }_{(0,10)}$ solution (the one which is "smeared" in $z$ direction) by formal $T$ duality in $t\left(A_{t} \rightarrow C\right.$, etc. $)$ and the identification of the dual $t$ coordinate with $i z$ ( $T$ duality in time direction transforms a real background in IIA theory into a complex one in type-IIB $(1,9)$ theory, but again a real background in typeIIB $_{(0,10)}$ theory). The consistency of this picture seems to suggest that, like the $\operatorname{SL}(2, Z)$ symmetry of type-IIB theory, the $T$ duality between type-IIB and type-IIA theories may have a simple origin in the 12-dimensional theory, being related to a coordinate transformation interchanging $y$ and $z$ compactification directions.

Finitely boosting 0-brane in one extra isometric direction $x_{9}$ [this corresponds to a wave along a generic cycle of 2-torus $\left(x_{11}, x_{9}\right)$ in $D=11$ theory [23]] and doing $T$ duality in $x_{9}$ [i.e., performing $\mathrm{O}(2,2)$ duality on the 0-brane background] leads to the $\operatorname{SL}(2, Z)$ family of strings [21] in type-IIB ${ }_{(1,9)}$ theory. Its counterpart in type-IIB ${ }_{(0,10)}$ theory, which may be interpreted as a mixture of a smeared instanton and a string, should correspond to a reduction of a 3-brane configuration (with nontrivial antisymmetric tensor background) in $(1,11)$ theory. [ $T$ duality between the smeared type IIB instanton and the 0-brane also implies the existence of a nonsupersymmetric (non-BPS) generalization of the smeared instanton (black instanton). Applying $T$ duality to the nonextremal 0 -brane (which is a dimensional reduction of the $D=11$ Schwarzschild background finitely boosted in an additional isometric 
direction, with the extremal case corresponding to the infinite boost limit), we find the following type-IIB ${ }_{(0,10)}$ solution [cf. (8)] $e^{\phi}=\hat{H}(x), C=\operatorname{coth} \beta\left[\hat{H}^{-1}(x)-1\right]$, $d s_{10 E}^{2}=f^{-1}(r)\left(d z^{2}+d r^{2}\right)+r^{2} d \Omega_{8}^{2}$, where $f=$ $1-\mu / r^{7}, \hat{H}=1+\hat{q} / r^{7}, \hat{q}=\mu \sinh ^{2} \beta=q \tanh \beta$, and $\mu$ and $\beta$ are the nonextremality and boost parameters. For $q=0$ this is $T$ dual to the $D=10$ Schwarzschild metric in the Euclidean time direction $(t=i z)$.]

The relation between the instanton charge and the 12-momentum also suggests an interpretation of instantons bound to Euclidean 3-brane world volume in typeIIB $_{(0,10)}$ theory [5] as corresponding to 3-branes boosted in the 12th dimension (cf. analogous 11-dimensional interpretation of 0-brane bound states in type-IIA theory [23,24]). [For a 3-brane probe in the $D$-instanton background one finds no nontrivial potential term in the 3-brane action. The 1/4 supersymmetric solution of type-IIB ${ }_{(0.10)}$ theory corresponding to a combination of a Euclidean 3-brane and an instanton is easy to construct explicitly; for example, the string-frame metric is $d s_{10}^{2}=H_{-1}^{1 / 2} H_{3}^{1 / 2}\left(H_{3}^{-1} d x_{k} d x_{k}+d x_{i} d x_{i}\right)$, where $k=$ $1, \ldots, 4, i=5, \ldots, 10$, and $H_{-1}$ and $H_{3}$ are the instanton and the 3-brane harmonic functions depending on $x_{i}$. In an alternative description, the instanton charge is generated by the gauge field instanton of the 3-brane worldvolume theory due to the presence of the $\int C F \wedge F$ coupling in the 3-brane action [25] (this may have a relation to a discussion of $D$-instantons in [26]). Finally, let us note that the above discussion of type-IIB ${ }_{(0,10)}$ theory, based on compactification of $D=12$ theory in one spatial and one timelike direction, suggests that a similar interpretation should also be possible for type-IIB ${ }_{(1,9)}$ theory: One is just to assume that the metric $g_{m n}$ in (6) has the Minkowski signature while still reducing in the $(t, y)$ directions. The $D=12$ theory then has the $(2,10)$ signature as in some of the proposals in [7,8]. Its 3-brane solution will then have to have the $(2,2)$ world-volume signature [to be related to type-IIB strings upon compactification in $(t, y)$ ] while the 5-brane may still have the usual $(1,5)$ signature.

I am very grateful to A. Schwimmer for a stimulating discussion. This work was supported by PPARC and the European Commission TMR programme ERBFMRXCT96-0045.

*Also at Lebedev Physics Institute, Moscow.

${ }^{\dagger}$ Electronic address: tseytlin@ic.ac.uk

[1] J.H. Schwarz, "The Second Superstring Revolution," lecture presented at the Sakharov Conference (Moscow, 1996), Report No. hep-th/9607067 (to be published); J. Polchinski, Rev. Mod. Phys. 68, 1245 (1996).
[2] M. B Green, J.H. Schwarz, and E. Witten, Superstring Theory, (Cambridge University Press, Cambridge, England, 1987), 2 Vols.

[3] E. Witten, Nucl. Phys. B443, 85 (1995).

[4] P. K. Townsend, Phys. Lett. B 350, 184 (1995); Phys. Lett. B 373, 68 (1996).

[5] J. H. Schwarz, Nucl. Phys. B226, 269 (1983); P. S. Howe and P. C. West, Nucl. Phys. B238, 181 (1984).

[6] C. M. Hull, Nucl. Phys. B468, 113 (1996).

[7] C. Vafa, Nucl. Phys. B469, 403 (1996).

[8] M. Blencowe and M. J. Duff, Nucl. Phys. B310, 387 (1988); H. Ooguri and C. Vafa, Nucl. Phys. B361, 469 (1991); Nucl. Phys. B367, 83 (1991); D. Kutasov and E. Martinec, Nucl. Phys. B477, 652 (1996); Report No. hep-th/9612102 (to be published); D. Kutasov, E. Martinec, and M. O' Loughlin, Nucl. Phys. B477, 675 (1996); A. A. Tseytlin, Nucl. Phys. B469, 51 (1996); D. Jatkar and K. Rama, Phys. Lett. B 388, 45 (1996); I. Bars, Phys. Rev. D 54, 5203 (1996); S. Hewson and M. J. Perry, Report No. hep-th/9612008 (to be published).

[9] J. Van Holten and A. Van Proeyen, J. Phys. A 15, 3763 (1982).

[10] T. Banks, W. Fischler, S. H. Shenker, and L. Susskind, Report No. hep-th/9610043 (to be published).

[11] J. Polchinski, Phys. Rev. Lett. 75, 4724 (1995); Report No. hep-th/9611050 (to be published).

[12] M. B. Green, Phys. Lett. B 266, 325 (1992); Phys. Lett. B 329, 435 (1994); Phys. Lett. B 354, 271 (1995); J. Polchinski, Phys. Rev. D 50, 6041 (1994).

[13] M. B. Green and M. Gutperle, Report No. hep-th/9612127 (to be published).

[14] N. Ishibashi, H. Kawai, Y. Kitazawa, and A. Tsuchiya, Report No. hep-th/9612115 (to be published).

[15] E. Witten, Nucl. Phys. B460, 335 (1996).

[16] V. Periwal, Report No. hep-th/9611103 (to be published).

[17] G. W. Gibbons, M. B. Green, and M. J. Perry, Phys. Lett. B 370, 37 (1996).

[18] S. S. Gubser, A. Hashimoto, I. R. Klebanov, and J. M. Maldacena, Nucl. Phys. B472, 231 (1996).

[19] E. Bergshoeff, C. Hull, and T. Ortín, Nucl. Phys. B451, 547 (1995); E. Bergshoeff, H. J. Boonstra, and T. Ortín, Phys. Rev. D 53, 7206 (1996).

[20] S. Ferrara, R. Minasian, and A. Sagnotti, Nucl. Phys. B474, 323 (1996).

[21] J. H. Schwarz, Phys. Lett. B 360, 13 (1995); 364, 252(E) (1995).

[22] B. R. Greene, A. Shapere, C. Vafa, and S. T. Yau, Nucl. Phys. B337, 1 (1990).

[23] J. G. Russo and A. A. Tseytlin, Report No. hepth/9611047 (to be published).

[24] M. R. Douglas, D. Kabat, P. Pouliot, and S. H. Shenker, Report No. hep-th/9608024 (to be published).

[25] M. Douglas, Report No. hep-th/9512077 (to be published).

[26] J.-S. Park, Report No. hep-th/9612096 (to be published). 